Yi Cheng ${ }^{1 *}$, Dongbei Sheng ${ }^{1 *}$, Xiangdong Li ${ }^{1+}$, Sumei Hong ${ }^{1}$, Lixia Guo' $^{1}$, Shasha Zhao ${ }^{1}$, Yue Yuan', Jingjing Xue ${ }^{1}$, Hui Tian1, Yipin Ren ${ }^{1}$, Wujie Liu $^{1 *}$ and Kegong Tian ${ }^{1,2 *}$

${ }^{I}$ National Research Center for Veterinary Medicine, High-Tech District, Luoyang, China

${ }^{2}$ College of Animal Science and Veterinary Medicine Henan Agricultural University, Zhengzhou, China \#These authors contributed equally

Dates: Received: 18 October, 2016; Accepted: 24 October, 2016; Published: 25 October, 2016

${ }^{*}$ Corresponding authors: Wujie Liu, National Research Center for Veterinary Medicine, Cuiwei Road, High-Tech District, Luoyang, China 471003, E-mail: jike008@126.com

Kegong Tian, College of Animal Science and Veterinary Medicine, Henan Agricultural University, Zhengzhou, China, Tel: + (86) 10-59198895; Fax: + (86) 10-59198899; E-mail: tiankg@263.net

www.peertechz.com

Keywords: Newcastle disease (ND); Inactivated vaccine; $\mathrm{HI}$ antibodies; Virus shedding

\section{Research Article \\ Efficacy of a Recombinant Genotype VII Vaccine against Challenge with Velogenic Newcastle Disease Virus}

\section{Abbreviations}

NDV: Newcastle Disease Virus; DPV: Days Post-Vaccination; DPC: Days Post-Challenge; $\mathrm{ELD}_{50}$ : Median Embryo Lethal Dose; $\mathrm{EID}_{50}: 50 \%$ Egg Infections Dose

\section{Introduction}

Newcastle virus (NDV) is an enveloped, nonsegmented singlestranded negative-sense RNA virus belonging to the genus Avulavirus within the family Paramyxoviridae [1]. NDVs are phylogenetically separated into two classes (I \& II) and further classified into different genotypes based on genetic differences [2]. Genotype VII viruses within class II that merged decades ago are now the major genotype of virus that are circulating in different countries including China [3]. The genome of NDV is approximately $15 \mathrm{~kb}$ and encodes 6 structural proteins including nucleoprotein (NP), phosphoprotein (P), matrix protein $(M)$, fusion protein $(F)$, hemagglutinin-neuraminidase $(H N)$, the large protein $(\mathrm{L})$, and two nonstructual proteins $\mathrm{V}$ and W. The NDV strains can be grouped as highly (velogenic), moderately (mesogenic), and weakly pathogenic (lentogenic) pathotypes according to cleavage site of fusion (F) gene which works as a virulence-determine gene [4].

Genotype VII NDV is a large and genetically diverse group of viruses which currently divided into 9 subgenotypes VIIa-VIIi [5]. All genotype VII viruses are predicted to be virulent with wide geographical distribution [1]. Commercial NDV vaccines including LaSota have been widely used in China decades ago. However, the sporadic outbreak of NDV genotype VII was reported which question the efficacy of commercial vaccines to the field viruses [6]. Also, the most widely used NDV vaccines including LaSota and B1 were
NDV genotype II strains [7]. The cross-protection of vaccines to genotype VII virus was seldom explored. In this study, we developed an inactivated NDV genotype VII vaccine based on reverse genetic techniques and compared its efficacy with commercial LaSota vaccine on SPF chickens.

\section{Material and methods}

\section{Virus}

NDV strain PLK-N-06 was isolated from an outbreak of ND in chickens and identified as velogenic [intracerebral pathogenicity index $(\mathrm{ICPI})=1.79$, mean death time $(\mathrm{MDT})=49 \mathrm{~h}$ ]. Phylogenetic analysis results showed that it belongs to NDV genotype VIId. The virus was grown in 10-day-old embryonated SPF chicken eggs. Allantoic fluid was collected for use.

\section{Construction of recombinant virus}

RNA was extracted from the virus with TRIzol reagent (Invitrogen, Carlsbad, CA, USA) according to the manufacturer's instructions. Seven pairs of primers were used to amplify full-length cDNA of PLK-N-06 by RT-PCR and then cloned into pCMVTNT plasmid as previously reported [8]. To attenuate PLK-N-06 virus, the $\mathrm{F}$ protein cleavage site was mutated with overlapping PCR by altering ${ }^{112} \mathrm{G}-\mathrm{R}-\mathrm{Q}-\mathrm{G}-\mathrm{R}-\mathrm{L}^{117}$ from ${ }^{112} \mathrm{R}-\mathrm{R}-\mathrm{Q}-\mathrm{K}-\mathrm{R}-\mathrm{F}^{117}$. The above constructed plasmid was next transfected with helper plasmids containing the individual NP, P, and L genes into BHK-21 cells using lipofectamine 2000 (Invitrogen, USA). After 3 days, the supernatant of cell culture containing the rescued virus was inoculated into allantoic cavities of SPF eggs. After 3 days, the allantoic fluid was harvested and the virus 
was detected by the hemagglutination (HA) test. The rescued virus was designated as N7a.

To check the stability of rescued virus, allantoic fluid containing virus was used to inoculate SPF eggs for ten consecutive times. RTPCR was performed to confirm the genetic stability of the mutated F gene.

\section{Vaccine efficacy study on chickens}

The attenuated N7a NDV was inactivated with $0.1 \%$ formaldehyde. The vaccine was prepared by emulsifying the above antigen solution with light mineral oils (MARCOL 52, France) at a ratio of 33:67 $(\mathrm{V} / \mathrm{V})$. The final dose of the inactivated vaccine contains $2 \times 10^{6} \mathrm{EID}_{50} /$ per bird in $20 \mu \mathrm{L}$. A commercial LaSota NDV vaccine (Dahuanong Biological Co. Ltd, China) was used according to the instruction of manufacture. Hemagglutination inhibition (HI) tests were performed by using LaSota and N7a antigen, the similarity of HN protein between these two strains of viruses was $90.2 \%$.

Forty 28-day old SPF chickens were divided into 4 groups with 10 chickens in each group. The maternal-derived HI antibodies were tested to be negative. Chickens in first two groups were vaccinated with $20 \mu \mathrm{L}$ experimental N7a vaccine or LaSota vaccine via intramuscular route. Chickens in next two groups were vaccinated with PBS as controls. After 28 days, the chickens in the first three groups were challenged with virulent PLK-N-06 NDV with $10^{5}$ $\mathrm{ELD}_{50}$ via intramuscular route. The chickens were kept for 14 days, and monitored daily for clinical signs and mortality. Chicken blood was collected to measure HI antibody titer at designated days. Oropharyogeal and cloacal swab samples were collected to isolate virus on chicken embryos and test the virus load by using EID $_{50}$. All chickens were kept in isolators and the animal experiments were approved by the Institutional Animal Care and Use Committee at National Research Center for Veterinary Medicine and conventional animal welfare regulations and standards were taken into account.

\section{Statistical analysis}

Statistical analysis was performed by one-way ANOVA test contained in the Prism 5.0 software package (GraphPad software Inc., San Diego, CA, USA), and a $p$ value of $\leq 0.05$ was considered statistically significant. Results are expressed as means and standard deviations.

\section{Results}

\section{Generation of recombinant N7a NDV}

The ICPI of PLK-N-06 NDV was 1.79 and belonged to velogenic strain. Due to the biosafety consideration, this virus has to be attenuated before applying as a vaccine. A cDNA clone encoding virus genome was synthesized by RT-PCR which was faithful copy of PLK-N-06 consensus sequence except for three amino acid changes in F protein. The mutated PLK-N-06 was designated as N7a and was recovered by transfection of BHK21 cells with cDNA plasmids and three helper plasmids. After 3 rounds of freeze-thaw cycles, the supernatant of cell culture was inoculated the embryonated chicken eggs. After 3 days, the recombinant N7a virus in the allantoic fluid was successfully rescued and confirmed by RT-PCR and sequencing (data not shown). The mutation in $\mathrm{F}$ gene was also confirmed by gene sequencing. The ICPI of N7a virus was 0.36 which belongs to lentogenic pathotype of NDV.

To characterize the rescued recombinant N7a virus, EID $_{50}$ and $\mathrm{HA}$ of $3^{\text {rd }}$ passages of virus were measured and compared with the parental virus strain. As shown in Figure 1A, the $\mathrm{EID}_{50}$ of recombinant virus was significantly higher than parental strain. Similarly, the HA titer of recombinant virus was also significantly higher than parental strain (Figure 1B). Therefore, the recombinant N7a NDV had a good proliferation on SPF embryos. To test the stability of recombinant virus, the $3^{\text {rd }}$ passages of virus was successively passages 10 times through SPF eggs, the introduced mutations in F gene was confirmed by RT-PCR which indicated the stability of the recombinant virus.

\section{Antibody response after vaccination}

Commercial NDV LaSota vaccine was used and compared with the experimental N7a vaccine as for the HI antibody response. The $\mathrm{HI}$ antibody titers to LaSota antigen and PLK-N-06 antigen were measured separately. As shown in Figure 2A, the HI titer of N7a to Lasota Ag was higher than the titer of LaSota, but the difference was not statistically significant. By contrast, N7a vaccine elicited earlier and significantly higher titer of HI titer to PLK-N-06 antigen at each time points as shown by Figure $2 \mathrm{~B}$. Therefore, vaccination of $\mathrm{N} 7$ a generated equivalent titer of $\mathrm{HI}$ antibodies to LaSota antigen but significantly higher $\mathrm{HI}$ antibodies to PLK-N-06 antigen which indicated N7a vaccine may have a better protection to NDV genotype VII infection.

\section{Protection to a velogenic NDV challenge}

At $28 \mathrm{dpv}$, the chickens in the first three groups were challenged with a velogenic NDV genotype VII strain PLK-N-06. The chickens

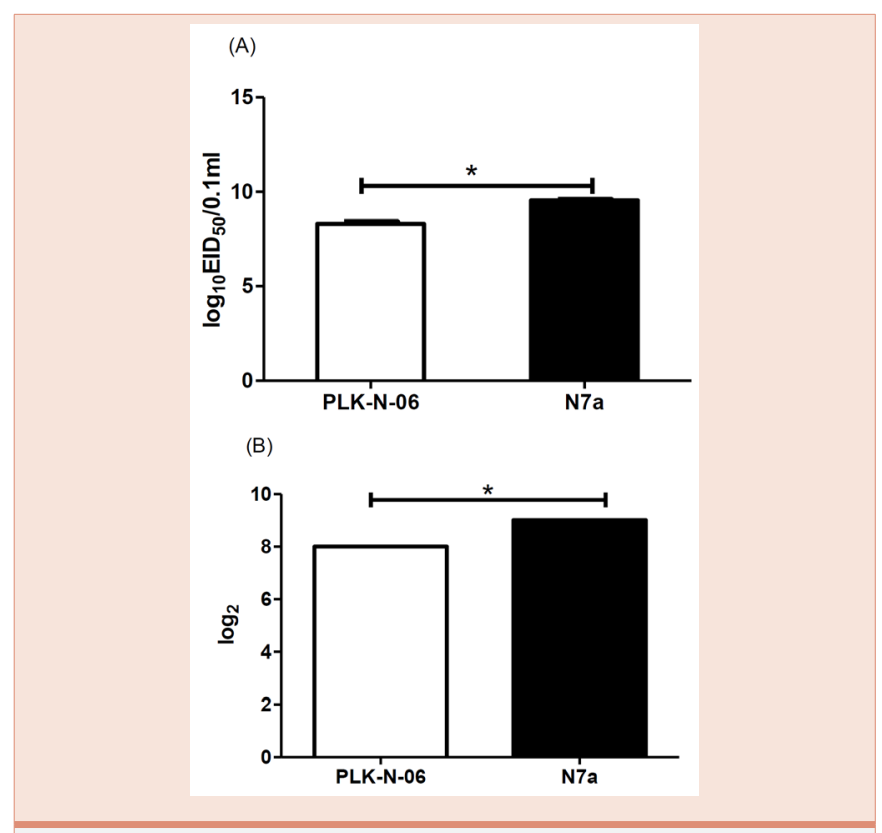

Figure 1: $E L D_{50}(A)$ and $H A(B)$ titers of recombinant N7a virus and its parental strain PLK-N-06. "“” indicates statistically significant $(p \leq 0.05)$. 
in the $4^{\text {th }}$ group worked as a sterile control throughout the study. All unvaccinated chickens displayed severe depression, respiratory distress and diarrhea from 3 to $4 \mathrm{dpc}$ and $100 \%$ mortality at $4 \mathrm{dpc}$ (Table 1). By contrast, the vaccinated chickens were completely protected without showing any clinical symptoms. At necropsy, hemorrhages were found in the glandular gastric mucosa, intestinal lymph nodes, and heart of unvaccinated chickens. No gross lesions were found in all vaccinated chickens and sterile control chickens which were euthanized at $14 \mathrm{dpc}$.

The shedding of challenge virus was examined from oropharyngeal and cloaca swabs at 3,5, and 7dpc. As shown in Table 1, virus was isolated from the cloacal swab samples of one LaSota-vaccinated chicken at $5 \mathrm{dpc}$ and one and two chickens in oropharyngeal and cloacal samples at $7 \mathrm{dpc}$, respectively. By contrast, there was no virus detected in N7a vaccinated chickens at above corresponding time points. As expected, no virus was detected in sterile control chickens. Therefore, the above data suggested N7a vaccine could provide complete protection to the velogenic NDV genotype VII challenge.

\section{Discussion}

The virulent NDV genotype VII has become the dominant genotype since 2000s in China [12] [9]. Among genotype VII, the
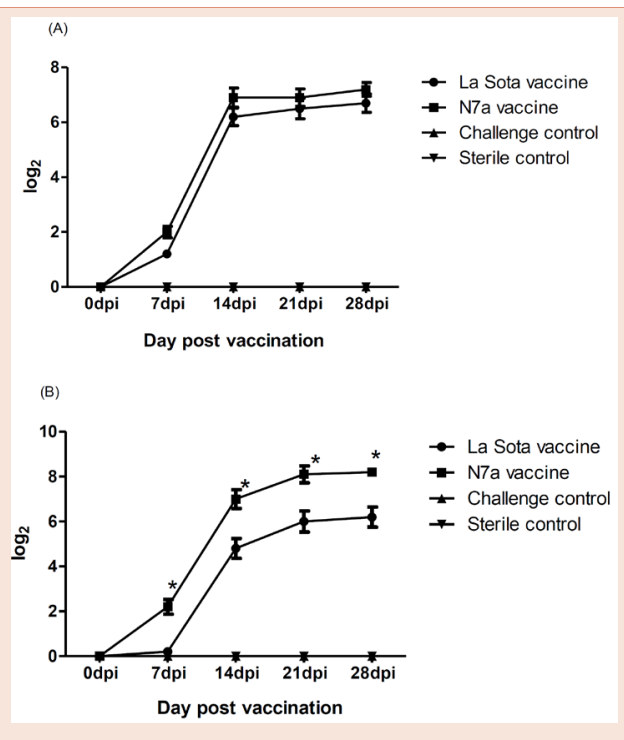

Figure 2: $\mathrm{HI}$ antibodies to LaSota antigen (A) and PLK-N-06 (B) after vaccination. sporadic outbreak of subgenotype VIId was recently reported [10]. The antigenic differences between the vaccine strain (exclusively genotype II) and circulating strain (mainly genotype VII) may contribute to the outbreaks of disease. Therefore, the new vaccines based on currently circulating virus strain are urgently needed to control the disease.

Several live attenuated NDV genotype VII vaccine were generated by using reverse genetics system, and vaccination on chickens showed good protection to the viral challenge [11]. However, there is a potential risk for the vaccine virus regaining its virulence during massive vaccination campaign in the field [12]. Also, virus shedding of the live vaccines was constantly reported [13]. Therefore, development of inactivated vaccine is necessary if it could provide good protection as live vaccine does but reduce virus shedding. In this study, we attenuated a virulent NDV genotype VII strain by using reverse genetic techniques and used it as an inactivated vaccine on SPF chickens. Compared with the commercial and previously reported vaccines, our experimental vaccine had the advantages of better performance of protection due to the higher HI titers without the risk of spread of vaccine viruses as the live vaccines did.

The recombinant N7a virus showed good proliferation ability on SPF eggs as the parental virus did (Figure 1). Both LaSota and $\mathrm{N} 7 \mathrm{a}$ antigens were used in the $\mathrm{HI}$ tests. $\mathrm{HI}$ antigen is related with $\mathrm{HN}$ protein of NDV and the similarity of HN protein between these two strains of viruses was $90.2 \%$. Compared to commercial LaSota vaccine, N7a vaccine elicited earlier and higher titers of $\mathrm{HI}$ antibodies to N7a antigen. This was consistent with previous reports that HI antibody titer can vary depending on homologous and heterologous antigens used for testing. And homologous antigens were normally found to elicited higher $\mathrm{HI}$ antibody titers compared to heterologous antigens [7]. The equivalent titer of $\mathrm{HI}$ antibody titer to LaSota and higher titer to PLK-N-06 indicated the N7a could work as a good vaccine candidate.

Since there were no clinical symptoms observed after virus challenge in both vaccinated groups, the challenge virus shedding worked as an important parameter to evaluate the efficacy of vaccines. Compared with commercial LaSota vaccine, there was no virus shedding in both oropharyngeal and cloacal samples after PLK-N-06 challenge in N7a vaccinated group which further confirmed the advantages of N7a working as a vaccine candidate to control NDV genotype VII infection.

Table 1: Frequency of isolation of challenge virus in chickens and virus titer $\left(E I D_{50}\right)$.

\begin{tabular}{|c|c|c|c|c|c|c|}
\hline \multirow{3}{*}{ Group } & \multicolumn{6}{|c|}{ Number of viral shedding chickens after PLK-N-06 challenge } \\
\hline & \multicolumn{2}{|c|}{$3 \mathrm{dpc}$} & \multicolumn{2}{|c|}{$5 \mathrm{dpc}$} & \multicolumn{2}{|c|}{$7 \mathrm{dpc}$} \\
\hline & oropharyngeal & cloacal & oropharyngeal & cloacal & oropharyngeal & cloacal \\
\hline LaSota vaccine & 0/10 & $0 / 10$ & $0 / 10$ & $1 / 1010^{1.25}$ & $\begin{array}{l}1 / 10 \\
10^{1.75}\end{array}$ & $\begin{array}{l}2 / 10 \\
10^{3.13 \#}\end{array}$ \\
\hline N7a vaccine & $0 / 10$ & $0 / 10$ & $0 / 10$ & $0 / 10$ & 0/10 & $0 / 10$ \\
\hline Challenge control & $10 / 10$ & $10 / 10$ & I & I & I & I \\
\hline Sterile control & $0 / 10$ & $0 / 10$ & $0 / 10$ & $0 / 10$ & $0 / 10$ & $0 / 10$ \\
\hline
\end{tabular}




\section{Conclusion}

To conclude, we prepared and evaluated an experimental inactivated NDV genotype VII vaccine on SPF chickens. The antibody response elicited by vaccine and results of virus challenge study indicated it could work as a good vaccine candidate to control the disease.

\section{Acknowledgment}

This work was supported by grant from Luoyang Heluo Talent Plan (Dr. Kegong Tian).

\section{Reference}

1. Dimitrov KM, Ramey AM, Qu X, Bahl J, Afonso CL (2016) Temporal, geographic, and host distribution of avian paramyxovirus 1 (Newcastle disease virus). Infect Genet Evol 39: 22-34.

2. Miller PJ, Afonso CL, Spackman E, Scott MA, Pedersen JC, et al. (2010) Evidence for a new avian paramyxovirus serotype 10 detected in rockhopper penguins from the Falkland Islands. J Virol 84: 11496-11504.

3. Wang Z, Liu H, Xu J, Bao J, Zheng D, Sun C, et al. (2006) Genotyping of Newcastle disease viruses isolated from 2002 to 2004 in China. Ann N Y Acad Sci 1081: 228-239.

4. Hu Z, Hu J, Hu S, Song Q, Ding P, et al. (2015) High levels of virus replication and an intense inflammatory response contribute to the severe pathology in lymphoid tissues caused by Newcastle disease virus genotype VIId. Arch Virol 160: 639-648.

5. Miller PJ, Haddas R, Simanov L, Lublin A, Rehmani SF, et al (2015) Identification of new sub-genotypes of virulent Newcastle disease virus with potential panzootic features. Infect Genet Evol 29: 216-229.
6. Huang Y, Yang S, Hu B, Xu C, Gao D, et al. (2015) Genetic, pathogenic and antigenic diversity of Newcastle disease viruses in Shandong Province, China. Veterinary microbiology 180: 237-244.

7. Roohani K, Tan SW, Yeap SK, Ideris A, Bejo MH, et al. (2015) Characterisation of genotype VII Newcastle disease virus (NDV) isolated from NDV vaccinated chickens, and the efficacy of LaSota and recombinant genotype VII vaccines against challenge with velogenic NDV. J Vet Sci 16: 447-457.

8. Yu Y, Qiu X, Xu D, Zhan Y, Meng C, et al. (2012) Rescue of virulent class I Newcastle disease virus variant 9a5b-D5C1. Virol J 9: 120.

9. Zhang S, Zhao L, Wang X, Zhang D, Zhao J, et al. (2011) Serologic and virologic survey for evidence of infection with velogenic Newcastle disease virus in Chinese duck farms. Avian diseases 55: 476-479.

10. Zhang YY, Shao MY, Yu XH, Zhao J, Zhang GZ (2014) Molecular characterization of chicken-derived genotype VIld Newcastle disease virus isolates in China during 2005-2012 reveals a new length in hemagglutininneuraminidase. Infect Genet Evol 21: 359-366.

11. Liu MM, Cheng JL, Yu XH, Qin ZM, Tian FL, et al. (2015) Generation by reverse genetics of an effective attenuated Newcastle disease virus vaccine based on a prevalent highly virulent Chinese strain. Biotechnol Lett 37: 12871296.

12. Hu S, Ma H, Wu Y, Liu W, Wang X, et al. (2009) A vaccine candidate of attenuated genotype VII Newcastle disease virus generated by reverse genetics. Vaccine 27: 904-910.

13. Kim SH, Xiao S, Collins PL, Samal SK (2016) LaSota fusion (F) cleavage motif-mediated fusion activity is affected by other regions of the $F$ protein from different genotype Newcastle disease virus in a chimeric virus: implication for virulence attenuation. J Gen Virol 97: 1-8. 